\title{
Sensitivity analysis in multi-criteria evaluation of the suitability of urban green spaces for recreational activities
}

\author{
Sercan Cakir ${ }^{\mathrm{a}}$ (corresponding author), Robert Hecht ${ }^{\mathrm{a}}$ and Kerstin Krellenberga,b \\ $\underline{\text { s.cakir@ioer.de, }}$ r.hecht@ioer.de, kerstin.krellenberg@univie.ac.at \\ ${ }^{a}$ Leibniz Institute of Ecological Urban and Regional Development, Dresden, Germany \\ ${ }^{\mathrm{b}}$ Department of Geography and Regional Research, Urban Studies Working Group, University of Vienna, Austria
}

\begin{abstract}
Urban green spaces can have potential positive impacts on climate, biodiversity, health, and generally on the quality of life for urban residents and are of great importance for pursuing recreational activities. People as urban green space users should therefore be well informed about where they are located within a city and what activities they are suitable for. As people's individual preferences play a significant role in the decision-making process for visiting urban green spaces, we present a method that enables to assess urban green spaces upon various activities in accordance with a range of criteria. A sensitivity analysis to investigate the influence of weighting the criteria in a multi-criteria evaluation of the suitability of urban green spaces for recreational activities depending on people's preferences is in the focus. Initial results are shown based on an exemplary use case of playing Frisbee. Overall, the study is embedded in the development of a spatial decision support system implemented in the form of a web app to assist urban residents make decisions in the context of green space use.
\end{abstract}

Keywords. sensitivity analysis, multi-criteria decision making, urban green space

\section{Introduction}

Cities are facing many challenges concerning the effects of ongoing global change and in providing their inhabitants with adequate living conditions under these circumstances. Urban green spaces such as public parks, playgrounds, but also brownfields and private gardens are fulfilling important functions to humans and the environment. For example, urban green spaces can have positive impacts on the urban micro climate and air quality (Bolund and Hunhammar, 1999), and the health of the population (Tost et al., 2019). They can also serve as places for experiencing nature, recreation, and social interaction (Dickinson and Hobbs, 2017; Krellenberg et al., 2014).

Urban green spaces are not evenly distributed in cities and do not show the same suitability for different activities such as jogging, biking, or reading, as this is based on users' needs in terms of the environmental affordances (Hadavi et al., 2015). What is more, existing map services often only provide information on the existence of urban green spaces without further explicit information about the existence of amenities and their suitability for certain activities, although publicly accessible urban green spaces and their amenities can now be automatically mapped using open and VGI data (Ludwig et al., 2021). Spatial decision support systems (SDSS) with their various approaches offer the possibility to evaluate the alternatives based on computed characteristics (Keenan and Jankowski, 2019) by integrating spatial data processing and multi-criteria decision making (MCDM) into a computer-based system. They are designed to enable people to make more efficient choices, especially when dealing with spatial decision-making issues (Malczewski, 1999). As, for example in the present case, to choose urban green spaces according to a range of weighted criteria under certain activities. SDSS can thus bring the information and available data together to support people in making their choice for the best suitable urban green spaces for their individual needs (in terms of criteria for pursuing certain activities). To better interpret the results of an SDSS, sensitivity analyses are used to examine the extent of output variation in a model when parameters are varied systematically over a certain range (Delgado and Sendra, 2004). Former studies on the assessment of urban green spaces using SDSS supported 
several research fields such as monitoring urban green spaces to aid local authorities for the enhancement of urban green spaces (Pelizaro et al., 2005), analyzing urban green spaces in terms of accessibility and quality to support sustainable urban planning (Stessens et al., 2017; Meng and Malczewski, 2015), and evaluating the suitability of sites for the further development of urban green spaces (Li et al., 2018). In this context, this paper presents a newly developed SDSS approach that allows the evaluation of urban green spaces in terms of their suitability upon a range of recreational activities.

As sensitivity analysis plays a significant role at the end of the SDSS development process and is explained as the investigation of how the uncertainties in the output of a model can be attributed to the uncertainties of its inputs (Saltelli, 2002), it is likewise applied. This refers, for example, to Grêt-Regamey et al. (2017) who developed an SDSS including a sensitivity analysis step to help allocate urban development areas, Chen et al. (2010) and Xu and Zhang (2013) who employed a method to better examine the sensitivity of criteria in the context of land suitability evaluation. Aminu et al. (2014) designed a system for sustainable tourism planning and employed a sensitivity analysis method to uncover the uncertainty of expert judgments given. Chen et al. (2018) proposed an MCDM method for a site selection problem followed by a sensitivity analysis step measuring the model sensitivity to changes in the criteria weights. Finally, the work of Myagmartseren et al. (2017) should be mentioned, who investigated the usability of GIS-based MCDM in terms of the development of new urban areas exploring the contribution of sensitivity analysis to their study. Our study, unlike existing approaches, examines the sensitivity of urban green space suitability to criteria weight variations in terms of undertaking several recreational activities in conjunction with developing an SDSS approach.

With all these in mind, the overall aim of the presented study is to ascertain both the influence of criteria weight simulation on urban green space suitability and the individual impacts of each criterion on the assessment of urban green spaces by enabling one of the most prominent sensitivity analysis methods, One-at-a-time (OAT) method (Chen et al., 2010; de Brito et al., 2019), in which the criteria weights in the developed SDSS approach are varied within a specific range. Therefore, this study seeks to address the following questions:

- What impacts does changing the criteria weights in the SDSS have on the assessment of urban green spaces?

- Given the association between activity and criteria, what are the predominant criteria that yield major impacts on urban green space suitability score calculation?
To this end, we first briefly present the developed SDSS approach with its steps and then address its linkage to the sensitivity analysis. We focus only on the presentation and discussion of the results of the implemented sensitivity analysis to assess the suitability of urban green spaces using an exemplary case study.

\section{Methodology}

Given the definition of SDSS (Malczewski, 1999), urban green spaces can be assessed with an SDSS approach in terms of their suitability for several activities by taking user preferences into account. As users expect a change in the results upon their personal preferences, the OAT sensitivity analysis method as a followup step investigates how sensitive the outcome is to different user preferences, which are the criteria weights.

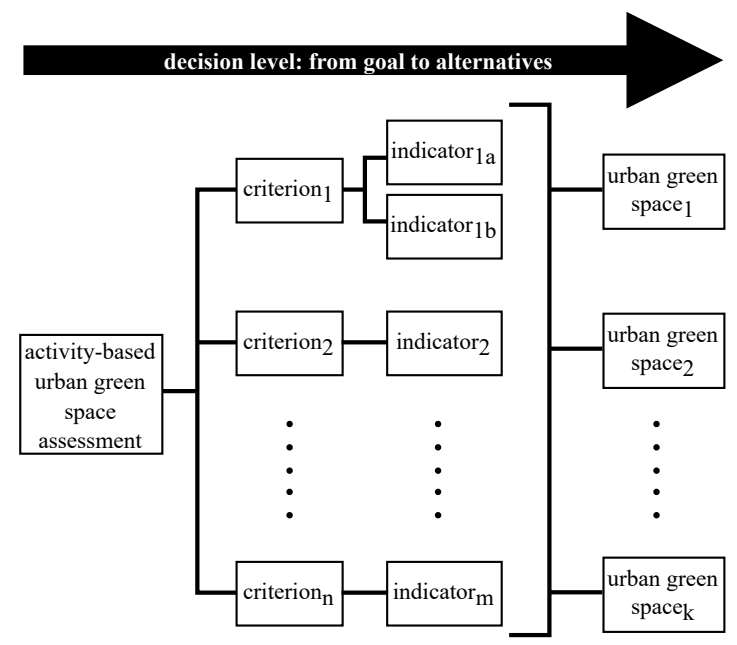

Figure 1. Hierarchy of multi-criteria decision making (adapted from Saaty (1990))

The developed SDSS approach for activity-based urban green space assessment comprises of the hierarchy of decision making (Fig. 1) provided by Saaty (1990) and calculation of criteria weights proposed by Keeney et al. (1993). Whilst forming a hierarchy helps us explicitly demonstrate the decision-making process from the objective to the assessment of urban green spaces, by including criteria and indicator definitions as intermediate steps, the calculation of criteria weights lead us to absorb the importance of user participation.

The steps of the developed SDSS approach are summarized in a workflow (Fig. 2). There are two preliminary steps: a) identify activities and their corresponding criteria, and b) calculate indicators to measure the degree of fulfillment in relation to the criterion. The results form the input for the five subsequent steps of the actual workflow (gray box): c) acquire user preferences for each criterion through two surveys (Krellenberg et al., 2021), d) calculate criteria weights via 
Multi-Attribute Utility Theory (MAUT) (Keeney et al., 1993), e) normalize indicator values, f) calculate suitability scores for urban green spaces, and finally g) apply sensitivity analysis to examine the sensitivity of urban green space suitability to changes in criteria weights. The results of the sensitivity analysis are denoted here with a dotted arrow as a potential input for the adaptation of the indicator set. A dashed arrow at the end of the workflow indicates that the developed approach is integrated into a web-based application. However, this article focuses on the implementation of sensitivity analysis and answering the mentioned research questions.

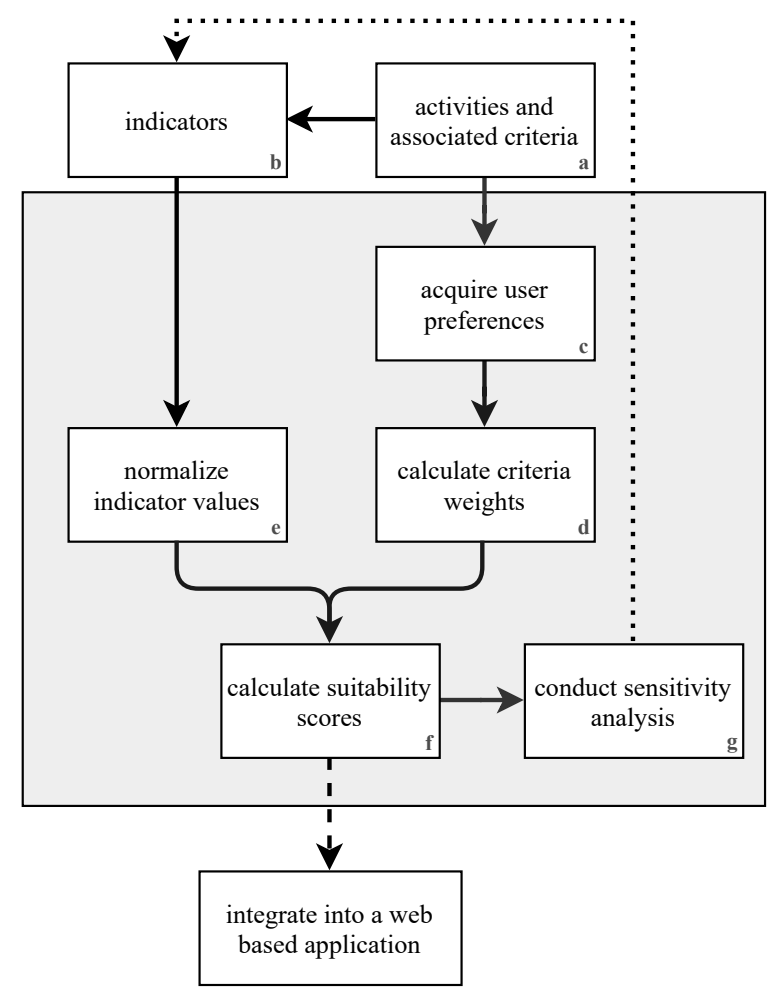

Figure 2. Overview of the workflow of the study composed of multiple steps from identification of activities to conducting sensitivity analysis

\subsection{Identification of activities and their corre- sponding criteria, and development of indica- tors}

The two preliminary steps in Fig. 2 are based on prior work by Krellenberg et al. (2021) and underlie our SDSS approach to evaluate the urban green spaces upon specific recreational activities. Thereby, relevant recreational activities and the corresponding criteria necessary for their practice were defined. For a spatial assessment of the criteria on the level of individual urban green spaces, indicators expressing the degree of fulfillment to a criterion in a numerical value were then developed and processed. A total of 18 activities (e.g. running, playing Frisbee) were identified and associ- ated with 34 criteria (e.g. meadow flatness) represented by 36 indicators (e.g. average slope of the largest continuous meadow area of the green space) (ibid.).

\subsection{Normalization of indicator values}

Indicator values were normalized in the range of $[0,1]$ according to individual utility functions (Keeney et al., 1993) for each criterion. They were defined by considering the nature of each criterion under the activities. For instance, the indicator values of meadow flatness under playing Frisbee were normalized using the negative min-max normalization (Ishizaka and Nemery, 2013), indicating that the flatter the meadow, the more suitable the urban green spaces.

\subsection{Acquisition of user preferences}

In this step, user preferences were obtained via importance scores for each criterion determining the weights. Through an on-site and an online survey, the participants were asked to assign an importance score to each criterion between not important at all and extremely important quantitatively recorded by values from 0 to 10 (Krellenberg et al., 2021). The results of these surveys were used for calculating the underlying criteria weights in the initial urban green space assessment, although the SDSS integrated into the web app includes a user preference acquisition interface to assess the urban green space suitability in real-time.

\subsection{Calculation of criteria weights}

The criteria weights were calculated as follows:

$W_{i}=\frac{P_{i}}{\sum_{i=1}^{n} P_{i}}$

$\sum_{i=1}^{n} W_{i}=1.0$

where $W_{i}$ and $P_{i}$ represent the calculated weight and the importance score assigned by the users for the $i$-th criterion, respectively. $n$ is the total number of criteria for the associated activity. Here the sum of the criteria weights for each activity must be equal to one.

\subsection{Calculation of suitability scores}

We designed the calculation of urban green space suitability scores as an activity-based approach. Once we acquired the importance scores for each criterion of a desired activity from the users, the criteria weights were calculated. Each urban green space was scored, 
possessing available data for the selected activity's criteria accordingly. The method carried out can be glanced in the following equation:

$S=\sum_{i=1}^{n} V_{i} \times W_{i}$

where $S$ is the calculated suitability score for the urban green spaces. $V_{i}$ and $W_{i}$ show respectively the normalized indicator value and weight of the $i$-th criterion.

\subsection{Sensitivity analysis}

We employed the OAT method to ascertain the sensitivity of the urban green space suitability against variations in criteria weights within the activity-based urban green space assessment. The simulation involves defining the criteria weight change range and the step size (Chen et al., 2010). Criteria weights were simulated in the range of $[-100 \%,+100 \%]$ with a step size of $20 \%$, and with a $-100 \%$ change within the criteria weights meant that the corresponding criterion is omitted from the urban green space suitability score calculation and $+100 \%$ results that the weight is doubled. Thereby we aimed at exploring the sensitivity of urban green space suitability at the outermost boundaries. In addition to Eq. (2) in which it was ensured that the sum of the underlying criteria weights equals one, the sum of each simulated criterion weight and their adjacent criteria weights must also be equal to one. Therefore, we modified the adjacent criteria weights according to the following equation proposed by Chen et al. (2010):

$$
\begin{array}{r}
w\left(c_{i}, \text { rate }\right)=\left(1-w\left(c_{m}, \text { rate }\right)\right) \times \frac{w\left(c_{i}, 0\right)}{1-w\left(c_{m}, 0\right)} \\
i \neq m, 1 \leqslant i \leqslant n
\end{array}
$$

where $w\left(c_{i}\right.$, rate $)$ indicates the adjacent criteria weights at a specific rate, $w\left(c_{m}\right.$, rate) shows the weight of the main changing criterion, $w\left(c_{i}, 0\right)$ and $w\left(c_{m}, 0\right)$ represent the underlying weights of the $i$-th criterion and main changing criterion, respectively. $n$ expresses the total number of criteria per activity.

To describe the sensitivity of urban green space suitability, we separated the urban green spaces based on their individual suitability scores into two groups namely low and high suitable using the Natural Breaks algorithm (Slocum et al., 2008). Thereafter, we observed the changes in the number of urban green spaces between these two suitability groups per simulation step. To ascribe meaning to these changes, we calculated a single change value between the number of urban green spaces in the base run and each simulation, and computed an average change value for each criterion with the following equations:

change $=\left(\frac{N_{\text {sim }}-N_{\text {base }}}{N_{\text {base }}}\right) \times 100$

where $N_{\text {sim }}$ and $N_{\text {base }}$ represent the number of urban green spaces per simulation and in the base run, respectively.

$a v g=\frac{\sum_{i=1}^{n} \mid \text { change }_{i} \mid}{n}$

where $n$ is the number of runs per criterion, which is ten in our case.

Finally, we will interpret the sensitivity of urban green space suitability to changes in criteria weights based on the calculated average values.

\section{Case study}

\subsection{Study area}

The city of Dresden, capital of the Free State of Saxony in Germany, serves as a study area for applying the SSDS approach. The population density in Dresden is 1616 inhabitants per $\mathrm{km}^{2}$ and the proportion of inhabitants living within a radius of $300 \mathrm{~m}$ and 700 $\mathrm{m}$ of urban green spaces that are respectively greater than 1 ha and 10 ha (Grunewald et al., 2019) is 60.2\% (i.e. urban green space accessibility), making the city $42^{\text {nd }}$ and $176^{\text {th }}$ places, respectively, out of 191 cities with at least 50,000 inhabitants in Germany (IOER, 2021). Thus, the information about the potential to undertake specific activities with regards to individual preferences can support the use among the population that not yet accessed the existing urban green spaces.

\subsection{Playing Frisbee as an example activity}

To exemplify the approach, we explored the sensitivity of urban green space suitability to changes in the criteria weights of the activity playing Frisbee. This activity can be done in many urban green spaces and there was a broad consensus among respondents of the conducted surveys about the criteria needed to play Frisbee. The top four criteria given by the respondents (Krellenberg et al., 2021) for playing Frisbee are shown in Tab. 1. The data set used contains a total of 2294 urban green space polygons with attributes of the respective indicators. The polygons were generated from a fusion of official city block geometry data, the public parks and green areas (opendata.dresden.de), 
and OpenStreetMap (www.openstreetmap.org). Detailed data sources of the indicators used can be found in Krellenberg et al. (2021).

Table 1. Activity playing Frisbee and its corresponding criteria and indicators

\begin{tabular}{l|l|l}
\hline activity & criteria & indicators \\
\hline & meadow size & $\begin{array}{l}\text { size of the largest } \\
\text { continuous meadow area } \\
\text { of the green space }\left[\mathrm{m}^{2}\right]\end{array}$ \\
\cline { 2 - 3 } $\begin{array}{l}\text { playing } \\
\text { Frisbee }\end{array}$ & shade & $\begin{array}{l}\text { share of tree area of total } \\
\text { green space }\left[\mathrm{m}^{2} / \mathrm{m}^{2}\right]\end{array}$ \\
\cline { 2 - 3 } & trees & $\begin{array}{l}\text { share of tree volume of total } \\
\text { green space }\left[\mathrm{m}^{3} / \mathrm{m}^{2}\right]\end{array}$ \\
\cline { 2 - 3 } & meadow flatness & $\begin{array}{l}\text { average slope of the largest } \\
\text { continuous meadow area of } \\
\text { the green space }\left[{ }^{\circ}\right]\end{array}$ \\
\hline
\end{tabular}

\section{Results}

In the case study Dresden, the simulation of criteria weights yielded ten different values per criterion for the activity playing Frisbee. These results are illustrated in Tab. 2 with an associated color gradient to visualize the impact of the step size in the simulation process. A red gradient is defined for negative weight changes and green for the positive ones. The base run is marked by gray colored lines. Uncolored columns represent the altered weights of the adjacent criteria as previously given in Eq. (4) with $w\left(c_{i}\right.$, rate $)$.

Using the base run criteria weights, the urban green spaces were scored to indicate how suitable they are for meeting the requirements of playing Frisbee. We used these scores to divide the urban green spaces into two groups as low and high suitable and illustrated the changes in the number of urban green spaces, that varied from the base run, as percentages per step. Additionally, to keep track of the average effect caused by the weight changes in the urban green space suitability, we calculated a mean urban green space score per step. Tab. 3 presents the results including the mean change in the number of urban green spaces with a green gradient for each criterion to indicate the sensitivity level of the urban green space suitability: the brighter the green, the higher the sensitivity, indicating that urban green space suitability for playing Frisbee is most sensitive to the criteria weight changes of meadow flatness with average values of $14.90 \%$ and $10.61 \%$ in the low and high group, respectively.

In addition to Tab. 3, we spatially mapped the suitability values of the urban green spaces in the city of Dresden (Fig. 3). The map shows the variations in suitability values for playing Frisbee using the default weights (middle) as well as the outermost limits of weight changes, which are $+100 \%$ (bottom) and $-100 \%$ (top). Suitability scores are shown from very
Table 2. Simulation of criteria weights for the activity playing Frisbee within the range of $[-100 \%,+100 \%]$

\begin{tabular}{|c|c|c|c|c|c|}
\hline & $\begin{array}{l}\text { weight } \\
\text { change }\end{array}$ & $\begin{array}{c}\text { meadow } \\
\text { size }\end{array}$ & shade & trees & $\begin{array}{c}\text { meadow } \\
\text { flatness }\end{array}$ \\
\hline \multirow{11}{*}{ 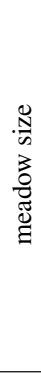 } & $-100 \%$ & 0.0000 & 0.3351 & 0.2984 & 0.3665 \\
\hline & $-80 \%$ & 0.0683 & 0.3122 & 0.2781 & 0.3415 \\
\hline & $-60 \%$ & 0.1366 & 0.2893 & 0.2577 & 0.3164 \\
\hline & $-40 \%$ & 0.2048 & 0.2664 & 0.2373 & 0.2914 \\
\hline & $-20 \%$ & 0.2731 & 0.2436 & 0.2169 & 0.2664 \\
\hline & base & 0.3414 & 0.2207 & 0.1966 & 0.2414 \\
\hline & $+20 \%$ & 0.4097 & 0.1978 & 0.1762 & 0.2164 \\
\hline & $+40 \%$ & 0.4779 & 0.1749 & 0.1558 & 0.1913 \\
\hline & $+60 \%$ & 0.5462 & 0.1521 & 0.1354 & 0.1663 \\
\hline & $+80 \%$ & 0.6145 & 0.1292 & 0.1150 & 0.1413 \\
\hline & $+100 \%$ & 0.6828 & 0.1063 & 0.0947 & 0.1163 \\
\hline \multirow{11}{*}{$\frac{\mathbb{Z}}{\tilde{J}}$} & $-100 \%$ & 0.4381 & 0.0000 & 0.2522 & 0.3097 \\
\hline & $-80 \%$ & 0.4187 & 0.0441 & 0.2411 & 0.2961 \\
\hline & $-60 \%$ & 0.3994 & 0.0883 & 0.2299 & 0.2824 \\
\hline & $-40 \%$ & 0.3800 & 0.1324 & 0.2188 & 0.2687 \\
\hline & $-20 \%$ & 0.3607 & 0.1766 & 0.2077 & 0.2551 \\
\hline & base & 0.3414 & 0.2207 & 0.1966 & 0.2414 \\
\hline & $+20 \%$ & 0.3220 & 0.2648 & 0.1854 & 0.2277 \\
\hline & $+40 \%$ & 0.3027 & 0.3090 & 0.1743 & 0.2140 \\
\hline & $+60 \%$ & 0.2834 & 0.3531 & 0.1632 & 0.2004 \\
\hline & $+80 \%$ & 0.2640 & 0.3972 & 0.1520 & 0.1867 \\
\hline & $+100 \%$ & 0.2447 & 0.4414 & 0.1409 & 0.1730 \\
\hline \multirow{11}{*}{$\underset{\Xi}{\Xi}$} & $-100 \%$ & 0.4249 & 0.2747 & 0.0000 & 0.3004 \\
\hline & $-80 \%$ & 0.4082 & 0.2639 & 0.0393 & 0.2886 \\
\hline & $-60 \%$ & 0.3915 & 0.2531 & 0.0786 & 0.2768 \\
\hline & $-40 \%$ & 0.3748 & 0.2423 & 0.1179 & 0.2650 \\
\hline & $-20 \%$ & 0.3581 & 0.2315 & 0.1572 & 0.2532 \\
\hline & base & 0.3414 & 0.2207 & 0.1966 & 0.2414 \\
\hline & $+20 \%$ & 0.3247 & 0.2099 & 0.2359 & 0.2296 \\
\hline & $+40 \%$ & 0.3080 & 0.1991 & 0.2752 & 0.2178 \\
\hline & $+60 \%$ & 0.2913 & 0.1883 & 0.3145 & 0.2059 \\
\hline & $+80 \%$ & 0.2746 & 0.1775 & 0.3538 & 0.1941 \\
\hline & $+100 \%$ & 0.2579 & 0.1667 & 0.3931 & 0.1823 \\
\hline \multirow{11}{*}{ 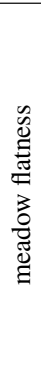 } & $-100 \%$ & 0.4500 & 0.2909 & 0.2591 & 0.0000 \\
\hline & $-80 \%$ & 0.4283 & 0.2769 & 0.2466 & 0.0483 \\
\hline & $-60 \%$ & 0.4066 & 0.2628 & 0.2341 & 0.0966 \\
\hline & $-40 \%$ & 0.3848 & 0.2488 & 0.2216 & 0.1448 \\
\hline & $-20 \%$ & 0.3631 & 0.2347 & 0.2091 & 0.1931 \\
\hline & base & 0.3414 & 0.2207 & 0.1966 & 0.2414 \\
\hline & $+20 \%$ & 0.3197 & 0.2066 & 0.1840 & 0.2897 \\
\hline & $+40 \%$ & 0.2979 & 0.1926 & 0.1715 & 0.3379 \\
\hline & $+60 \%$ & 0.2762 & 0.1786 & 0.1590 & 0.3862 \\
\hline & $+80 \%$ & 0.2545 & 0.1645 & 0.1465 & 0.4345 \\
\hline & $+100 \%$ & 0.2328 & 0.1505 & 0.1340 & 0.4828 \\
\hline
\end{tabular}

light (minimum) to dark green (maximum). Noticeable are the large urban green spaces adjacent to each other, located towards the northeast of the city. These belong to the Dresden Heath, which is a forestry area in Dresden and important for recreational activities.

\section{Discussion}

Our findings related to the first research question indicate that even small changes in criterion weights strongly affect the scores of urban green spaces for the example activity playing Frisbee. Therefore, the suitability of urban green spaces for the chosen activity is highly influenced by user preferences. 
Table 3. Sensitivity of urban green space (UGS) suitability to the criteria weight simulation

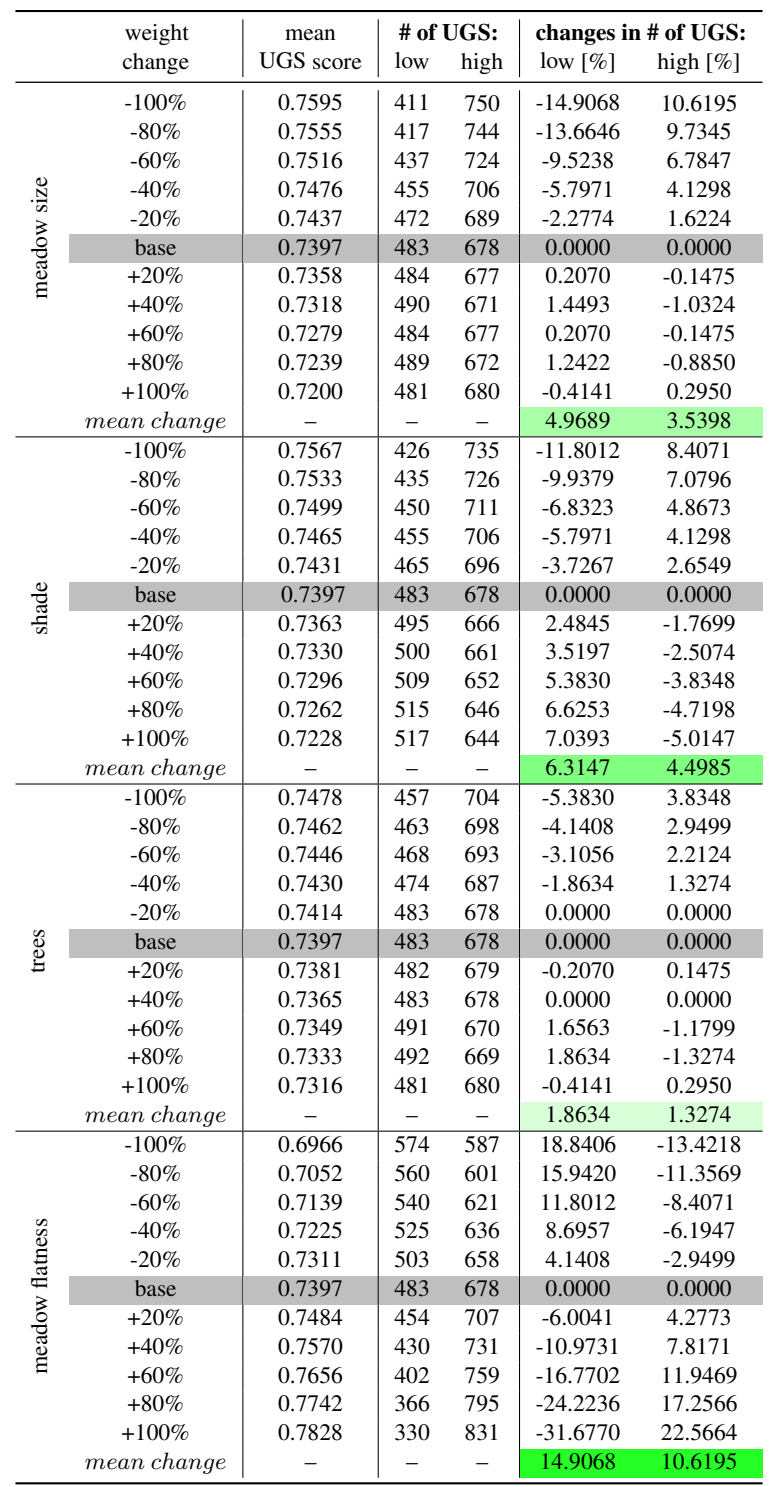

Considering the second research question, we investigated which criteria the suitability of urban green spaces is most sensitive to. Myagmartseren et al. (2017) concluded that the highest number of changes between different suitability classes occur when the most important criterion in the base run is simulated. However, our results show that the changes are independent of the underlying criteria weights. That is to say, the most important criterion in the base run is not necessarily the criterion that urban green space suitability is the most sensitive to. Based on the underlying values, meadow size is the most important criterion in our study. However, considering the results of sensitivity analysis, the urban green space suitability is most sensitive to the simulation of weights of meadow flatness, which is the second most important criterion in the base run. The reason here could be other steps of the workflow (Fig. 2), e.g. normalization procedure for

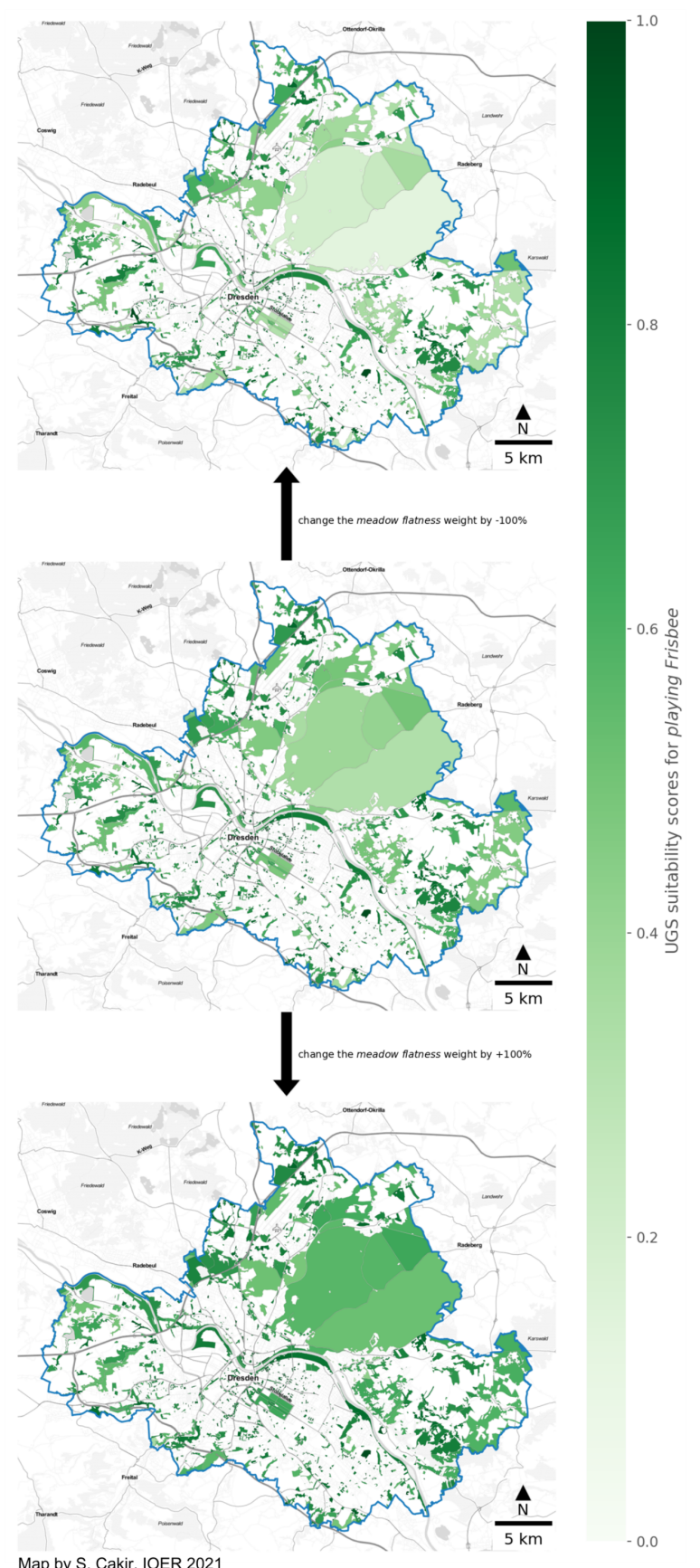

Map by S. Cakir, IOER 2021

Basemap tiles by Stamen Design, under CC BY 3.0

Basemap data by OpenStreetMap contributers, under ODbL

Figure 3. Illustration of urban green space suitability in the city of Dresden upon playing Frisbee with the base meadow flatness weight (middle), decreased weight by $100 \%$ (top), and increased weight by $+100 \%$ (bottom)

indicator values. Compared to meadow flatness, where an average 72 urban green spaces moved between two suitability classes in the analysis for the City of Dresden, urban green space suitability is least sensitive to changes of trees weights, where only 9 of the urban green spaces moved between two classes (Tab. 3).

Additionally, the mean urban green space scores in Tab. 3 are not entirely consistent with the changes in 
the number of urban green spaces. For instance, a 20\% negative change in the weight of trees increases the mean urban green space score by about $0.23 \%$, whilst it does not make any difference in the change in the number of urban green spaces. This could mean that this much change in the weights still affects the urban green space score calculation, whereas its effect does not necessarily be the same as the changes in the number of urban green spaces between two suitability classes. However, this could be made more consistent by dividing the urban green spaces into more than two suitability classes and then allowing smaller gradual changes to be considered.

The results suggest that users can expect a greater change in urban green space suitability when different importance scores are assigned to meadow flatness than to other criteria. This underpins that the predominant criterion for undertaking playing Frisbee in Dresden is the meadow flatness. Therefore, an accurate measurement of this criterion must be ensured by the developers of the web app to provide feasible and practical results for the users.

Whilst interpreting the results of the sensitivity analysis, uncertainties associated with the sample size also need to be taken into account. For example, if a criterion is highly sensitive, special attention should be paid to ensuring high quality of survey-based results on the importance scores obtained for that criterion (see Krellenberg et al. (2021)), which increases with the number of responses (large sample). The more sensitive a criterion, the more accurate the measurement instrument should be to determine the default weights for the different activities. Furthermore, in case certain criteria are of very high sensitivity, an attempt could be made to use this information to more precisely define related activities in order to provide adequate default weights for more specific user groups (e.g., for beginner Frisbee players and professional). This means that default weights can be more adequately captured the better the knowledge of the sensitivity of the system.

\section{Conclusion and Outlook}

Investigating the sensitivity of the urban green space suitability for undertaking recreational activities provides important findings to uncover the impacts of the user-indicated preferences. By simulating these preferences, which are the criteria weights, around a specific range in the multi-criteria evaluation of urban green spaces, this study demonstrated that each criterion has a distinctive influence on the urban green space suitability for one defined recreational activity: playing Frisbee. Our method can simply be applied to other types of activities to help deduce the predominant criteria in the urban green space assessment.
Our sensitivity analysis approach considers only the simulation of user-dependent inputs and does not focus on simulating the indicator values, which can also potentially affect the urban green space assessment. Future research, implementing the sensitivity analysis from a developer's perspective, should aim to examine not only the impact of criteria weights simulation but also the indicator values and their calculation methods. Moreover, investigating the sensitivity of urban green space suitability by varying multiple weights simultaneously rather than just one of them could be a good future approach to address the research question in more detail.

\section{Software and data availability}

To be able to perform the described procedure, multiple functions are developed in Python programming language using various packages such as Pandas, GeoPandas, and NumPy. The data sets used in this study are two GeoJSON files including the city boundary and the urban green space geometries with the associated indicator values for the selected activity. All the data and source code related to this study are accessible at doi.org/10.5281/zenodo.4672538 with instructions included in the README.md.

\section{References}

Aminu, M., Matori, A. N., and Yusof, K. W.: A spatial decision support system (SDSS) for sustainable tourism planning in Cameron Highlands, Malaysia, IOP Conference Series: Earth and Environmental Science, 18, 012 139, https: //doi.org/10.1088/1755-1315/18/1/012139, 2014.

Bolund, P. and Hunhammar, S.: Ecosystem services in urban areas, Ecological Economics, 29, 293-301, https://doi. org/10.1016/S0921-8009(99)00013-0, 1999.

Chen, J., Wang, J., Baležentis, T., Zagurskaite, F., Streimikiene, D., and Makutenienè, D.: Multicriteria approach towards the sustainable selection of a teahouse location with sensitivity analysis, Sustainability, 10, 2926, https: //doi.org/10.3390/su10082926, 2018.

Chen, Y., Yu, J., and Khan, S.: Spatial sensitivity analysis of multi-criteria weights in GIS-based land suitability evaluation, Environmental Modelling Software, 25, 1582-1591, https://doi.org/10.1016/j.envsoft.2010.06.001, 2010.

de Brito, M. M., Almoradie, A., and Evers, M.: Spatiallyexplicit sensitivity and uncertainty analysis in a MCDAbased flood vulnerability model, International Journal of Geographical Information Science, 33, 1788-1806, https://doi. org/10.1080/13658816.2019.1599125, 2019.

Delgado, M. G. and Sendra, J. B.: Sensitivity Analysis in Multicriteria Spatial Decision-Making: A Review, Human and Ecological Risk Assessment: An International Journal, 10, 1173-1187, https://doi.org/10.1080/ $10807030490887221,2004$. 
Dickinson, D. C. and Hobbs, R. J.: Cultural ecosystem services: Characteristics, challenges and lessons for urban green space research, Ecosystem Services, 25, 179-194, https:// doi.org/10.1016/j.ecoser.2017.04.014, 2017.

Grunewald, K., Richter, B., and Behnisch, M.: MultiIndicator Approach for Characterising Urban Green Space Provision at City and City-District Level in Germany, International Journal of Environmental Research and Public Health, 16, https://doi.org/10.3390/ijerph16132300, 2019.

Grêt-Regamey, A., Altwegg, J., Sirén, E. A., van Strien, M. J., and Weibel, B.: Integrating ecosystem services into spatial planning - A spatial decision support tool, Landscape and Urban Planning, 165, 206 - 219, https://doi.org/ 10.1016/j.landurbplan.2016.05.003, 2017.

Hadavi, S., Kaplan, R., and Hunter, M. C. R.: Environmental affordances: A practical approach for design of nearby outdoor settings in urban residential areas, Landscape and Urban Planning, 134, 19-32, https://doi.org/10.1016/j.landurbplan. 2014.10.001, 2015.

IOER: Monitor of Settlement and Open Space Development; editing status 2021-03-17; re3data.org - Registry of Research Data Repositories, https://doi.org/10.17616/R3QF5P, last accessed: 2021-03-27, 2021.

Ishizaka, A. and Nemery, P.: Multi-Criteria Decision Analysis: Methods and Software, John Wiley \& Sons, Ltd, https: //doi.org/10.1002/9781118644898, 2013.

Keenan, P. B. and Jankowski, P.: Spatial Decision Support Systems: Three decades on, Decision Support Systems, 116, 64-76, https://doi.org/10.1016/j.dss.2018.10.010, 2019.

Keeney, R. L., Raiffa, H., et al.: Decisions with multiple objectives: preferences and value trade-offs, Cambridge university press, https://doi.org/10.1017/CBO9781139174084, 1993.

Krellenberg, K., Welz, J., and Reyes-Päcke, S.: Urban green areas and their potential for social interaction - A case study of a socio-economically mixed neighbourhood in Santiago de Chile, Habitat International, 44, 11-21, https://doi.org/10. 1016/j.habitatint.2014.04.004, 2014.

Krellenberg, K., Artmann, M., Stanley, C., and Hecht, R.: What to do in, and what to expect from, urban green spacesIndicator-based approach to assess cultural ecosystem services, Urban Forestry \& Urban Greening, https://doi.org/10. 1016/j.ufug.2021.126986, 2021.

Li, Z., Fan, Z., and Shen, S.: Urban green space suitability evaluation based on the AHP-CV combined weight method: A case study of Fuping County, China, Sustainability, 10, 2656, https://doi.org/10.3390/su10082656, 2018.

Ludwig, C., Hecht, R., Lautenbach, S., Schorcht, M., and Zipf, A.: Mapping Public Urban Green Spaces Based on OpenStreetMap and Sentinel-2 Imagery Using Belief Functions, ISPRS International Journal of Geo-Information, 10, https://doi.org/10.3390/ijgi10040251, 2021.

Malczewski, J.: GIS and multicriteria decision analysis, John Wiley \& Sons, 1999.

Meng, Y. and Malczewski, J.: A GIS-based multicriteria decision making approach for evaluating accessibility to public parks in Calgary, Alberta, Human Geographies, 9, 29, https://doi.org/10.5719/hgeo.2015.91.3, 2015.
Myagmartseren, P., Buyandelger, M., and Brandt, S. A.: Implications of a spatial multicriteria decision analysis for urban development in Ulaanbaatar, Mongolia, Mathematical Problems in Engineering, 2017, https://doi.org/10.1155/ 2017/2819795, 2017.

Pelizaro, C., Arentze, T., and Timmermans, H.: A spatial decision support system for the provision and monitoring of urban greenspace, Technische Universiteit Eindhoven, Faculteit Bouwkunde, https://doi.org/10.6100/IR594825, 2005.

Saaty, T. L.: How to make a decision: The analytic hierarchy process, European Journal of Operational Research, 48, 926, https://doi.org/10.1016/0377-2217(90)90057-I, desicion making by the analytic hierarchy process: Theory and applications, 1990.

Saltelli, A.: Sensitivity analysis for importance assessment, Risk analysis, 22, 579-590, https://doi.org/10.1111/ 0272-4332.00040, 2002.

Slocum, T., McMaster, R., Kessler, F., Howard, H., and Mc Master, R.: Thematic Cartography and Geographic Visualization, Prentice Hall, 3rd edn., 2008.

Stessens, P., Khan, A. Z., Huysmans, M., and Canters, F.: Analysing urban green space accessibility and quality: A GIS-based model as spatial decision support for urban ecosystem services in Brussels, Ecosystem services, 28, 328-340, https://doi.org/10.1016/j.ecoser.2017.10.016, 2017.

Tost, H., Reichert, M., Braun, U., Reinhard, I., Peters, R., Lautenbach, S., Hoell, A., Schwarz, E., Ebner-Priemer, U., Zipf, A., et al.: Neural correlates of individual differences in affective benefit of real-life urban green space exposure, Nature Neuroscience, 22, 1389-1393, https://doi.org/10.1038/ s41593-019-0451-y, 2019.

$\mathrm{Xu}$, E. and Zhang, H.: Spatially-explicit sensitivity analysis for land suitability evaluation, Applied Geography, 45, 1-9, https://doi.org/10.1016/j.apgeog.2013.08.005, 2013. 